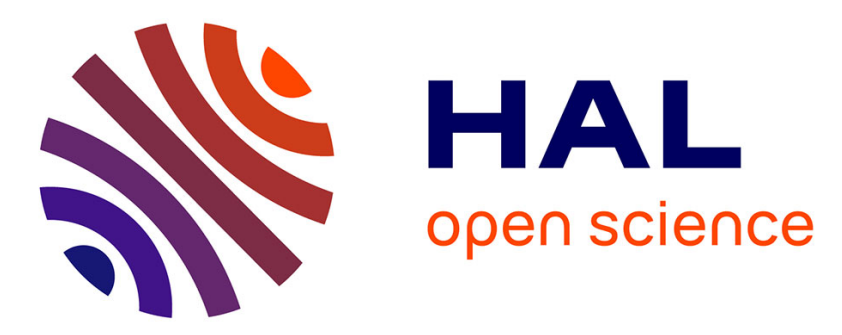

\title{
Une évaluation de la taille de l'économie informelle par un système complet de demande estimé sur données monétaires et temporelles
}

Armagan Tuna Aktuna-Gunes, Christophe Starzec, François Gardes

\section{To cite this version:}

Armagan Tuna Aktuna-Gunes, Christophe Starzec, François Gardes. Une évaluation de la taille de l'économie informelle par un système complet de demande estimé sur données monétaires et temporelles. Revue Economique, 2014, Avancées de la Recherche en Microéconomie Appliquée à l'occasion des XXX JMA (Nice 2013), 65 (4), pp.567. hal-01307146

\section{HAL Id: hal-01307146 \\ https://hal.science/hal-01307146}

Submitted on 26 Apr 2016

HAL is a multi-disciplinary open access archive for the deposit and dissemination of scientific research documents, whether they are published or not. The documents may come from teaching and research institutions in France or abroad, or from public or private research centers.
L'archive ouverte pluridisciplinaire HAL, est destinée au dépôt et à la diffusion de documents scientifiques de niveau recherche, publiés ou non, émanant des établissements d'enseignement et de recherche français ou étrangers, des laboratoires publics ou privés. 


\title{
Une évaluation de la taille de l'économie informelle par un système complet de demande estimé sur données monétaires et temporelles
}

\author{
Armagan T. Aktuna-Gunes* \\ Christophe Starzec** \\ François Gardes*
}

\begin{abstract}
Afin d'estimer la taille de l'économie informelle en Turquie, nous estimons un système de demande basé sur l'analyse du comportement de consommation proposée par Pissarides, Weber [1989], Lyssiotou et al. [2004] et Fortin et al. [2009]. Cette estimation est effectuée sur les dépenses monétaires et sur la somme des dépenses monétaires et temporelles (consacrées aux activités domestiques des ménages). Les informations nécessaires sur les inputs monétaires et temporels dans les dépenses de consommation des ménages sont obtenues par l'appariement statistique des enquêtes turques sur le Budget des familles avec l'enquête sur l'Emploi du temps [2006]. Comme prévu, la taille de l'économie informelle en Turquie estimée en utilisant les dépenses complètes (temps plus monnaie) est plus élevée que celles obtenues par l'approche des dépenses monétaires (respectivement $40,6 \%$ et $33,5 \%$ du pib pour les travailleurs indépendants et $20,7 \%$ et $14,1 \%$ du pib pour les salariés) et également plus élevée que celle obtenue par les méthodes macroéconomiques plus conventionnelles (35,1\%).
\end{abstract}

\section{A NEW ESTIMATION OF THE SIZE OF INFORMAL ECONOMY USING MONETARY AND FULL EXPENDITURES IN A COMPLETE DEMAND SYSTEM}

We use the demand system approach to estimate the size of informal economy in Turkey following the methodology based on the analysis of the individual consumption behaviour proposed by Pissarides, Weber [1989], Lyssiotou et al. [2004] and Fortin et al. [2009]. We extend this method by taking into account both the monetary expenditures and time spent on domestic activities. The necessary

* Paris School of Economics, Université Paris 1 Panthéon-Sorbonne, Centre d'économie de la Sorbonne. Correspondance : Centre d'économie de la Sorbonne, 106-112 boulevard de l'Hôpital, 75647, Paris Cedex 13, France.Courriel : Armagan.Aktuna-Gunes@univ-paris1.fr, Francois. Gardes@univ-paris1.fr

** Paris School of Economics, CNRS-CES. Correspondance : Centre d'Économie de la Sorbonne, 106-112 boulevard de 1'Hôpital, 75647, Paris Cedex 13, France. Courriel : christophe. starzec@univ-paris1.fr

Cette recherche a été financée par le contrat ANR MALDI et présentée aux colloques suivants : $\mathrm{XXX}^{\mathrm{es}}$ Journées de microéconomie appliquée, Nice, $2013 ; 62^{\mathrm{e}}$ congrès de 1'AFSE, Aix-en-Provence et $28^{\mathrm{e}}$ congrès de la European Economic Association, Gothenburg, 2013. Nous remercions les rapporteurs de cet article et les discutants à ces colloques. 
information of money and time inputs in consumption on the household's level is obtained by a statistical match of the Turkish Family Budget and Time Use surveys [2006]. As expected, the estimated model size of the informal economy in Turkey using the full (time plus money) expenditure is higher than those obtained by only monetary expenditure approach (in average $40.6 \%$ and $33.5 \%$ of GDP for self employers and $20.7 \%$ and $14.1 \%$ of GDP for wage-earners respectively) and also higher than that obtained by more conventional macroeconomic methods (35.1\%).

Classification JEL : D01, D12, E26, C81

\section{INTRODUCTION}

On considère généralement que les activités économiques informelles s'expliquent par le double motif d'éviter la taxation et d'améliorer son niveau de vie (voir Schneider et Enste [2000]). L'identification des marchés noirs et de ses mécanismes, qui constitue un objectif essentiel des autorités publiques, est rendue difficile par le manque de statistiques fiables et précises. Les méthodes les plus utilisées pour évaluer la taille de l'économie informelle se basent sur une approche macroéconomique, mais elles fournissent des évaluations disparates (voir Schneider et Enste [2000]), ce qui ne facilite pas la mise en œuvre de politiques appropriées. Dans le cas de la Turquie, les méthodes d'évaluation utilisées sont fondées sur la demande de monnaie (Ogunc-Yilmaz [2000] ; Cetintas-Vergil [2003]), la perception des impôts (Ilgın [2002]), l'utilisation d'électricité (Us [2004]) et la méthode Dynamic Multiple Indicators Multiple Causes (DYMIMIC, Schneider-Savasan [2007]). La multiplicité des évaluations obtenues a amené Ulgen et Ozturk [2006] à en discuter la fiabilité : l'estimation de la taille de l'économie informelle varie en effet selon les méthodes, pour des périodes récentes comparables, de 3,61 à 139\% de l'économie officielle (Akalin et Kesikoglu [2007]).

Les hypothèses qui permettent d'effectuer ces estimations macroéconomiques sont très critiquées : Thomas [1999] note par exemple leur absence de fondements théoriques, ce qui donne de l'intérêt aux rares études fondées sur une approche microéconomique. Deux méthodes microéconomiques ont été proposées : la première se base sur des enquêtes directes auprès de la population (Feinstein [1999]), tandis que la seconde ("the expenditure-based ", méthode de Pissarides et Weber [1989]) cherche à révéler le déficit de report par l'observation de la consommation alimentaire. Cette approche a été utilisée pour la Turquie par Davutyan [2008] dont l'évaluation de l'économie informelle s'établit à $21 \%$ en 2005.

Nous suivons, dans cet article, l'approche par les systèmes complets de demande initiée par Lyssiotou et al. [2004] (voir également une application au Québec de Fortin et al. [2009])1. L'évaluation de l'économie informelle,

1. Selon Lyssiotou et al. [2004], l'économie souterraine représente en Grande-Bretagne, dans l'évaluation basée sur l'estimation d'un système complet de demande, 10,6\% du PNB. L'évaluation au Québec se situe entre 4,6 et 5,7 \% en 1997-2002 (Fortin et al. [2009]). 
opérée sur les données individuelles de quatre enquêtes successive de 2003 à 2006, s'opère par l'estimation du sous-report de revenu, qui est identifiée par comparaison avec la dépense alimentaire supposée reportée de manière exacte, de même que les salaires des membres du ménage (la taxe sur les salaires étant opérée à la source, seuls les revenus au noir risquent de ne pas être reportés) : la propension marginale à consommer est donc supposée identique pour les composantes déclarée et non déclarée des revenus. Le report inexact des revenus concerne donc, dans notre étude, les employeurs individuels indépendants dont on compare revenus déclarés et consommation à ceux des salariés d'entreprises. La taille de l'économie informelle sera alors estimée en tenant compte de la proportion des travailleurs indépendants dans la production nationale.

Par rapport aux articles de Lyssiotou et al. [2004], Fortin et al. [2009], notre contribution concerne :

i. L'utilisation de données composant les dépenses monétaires avec le temps passé aux diverses activités (obtenues par un appariement d'une enquête de Budget de famille avec une enquête de Budget-temps, voir Gardes [2014]). Cette extension permet d'apprécier dans quelle mesure la contrainte temporelle influence l'extension du travail au noir.

ii. Le modèle fondé sur l'estimation d'un système de demande est appliqué pour la première fois à un pays en développement. L'importance des marchés informels dans ce type de pays devrait faire dépendre plus fortement la substitution de revenus formel et informel de la production domestique ${ }^{2}$.

iii. L'estimation du déficit de report des revenus concerne dans nos estimations les salariés aussi bien que les travailleurs indépendants.

On commence par présenter le modèle théorique en considérant plusieurs sources possibles du report imparfait des revenus dont les limites sont discutées ; la spécification économétrique est alors introduite ainsi que les données monétaires et temporelles et la méthode d'appariement statistique des deux types d'enquête. L'article se termine par la discussion des résultats et des évaluations de l'économie informelle qui s'en déduisent.

\section{MODĖLE THÉORIQUE}

Lyssiotou et al. [2004] et Fortin et al. [2009] considèrent des ménages munis de préférences séparables entre biens durables et non durables représentées par une fonction de coût : $\mathrm{C}(\mathbf{p}, \mathrm{U})=\mathrm{F}(c(\mathbf{p}, \mathrm{U}), d(\mathbf{r}, \mathrm{U}), \mathrm{U})$, où $\mathbf{p}, \mathbf{r}$ et $\mathrm{U}$ indiquent les vecteurs des prix de biens non durables et durables et le niveau d'utilité atteint par le ménage, les fonctions $c($.$) et d($.) représentant les indices agrégés des prix pour ces mêmes biens. Ces indices peuvent donc être interprétés comme des fonctions de coût qui reflètent les coûts unitaires payés par les ménages pour ces deux types de biens. Chacune de ces fonctions est croissante en $U$ et homogène et

2. Les activités domestiques jouent un rôle plus important dans les pays en développement, ce qui influence la taille de l'économie informelle. C'est la raison pour laquelle la prise en compte du temps peut modifier sensiblement l'évaluation de cette taille. 
linéaire dans les prix. Cette structure permet d'écrire le choix de consommation du ménage comme un processus à deux étapes :

a. Le ménage commence à allouer son revenu $\mathrm{Y}^{*}$ aux dépenses en biens durables et non durables selon la fonction de coût $\mathrm{C}$ (cette optimisation dépend donc des fonctions agrégatrices $c($.$) et d()$.$) .$

Par exemple, la demande pour le $i^{e ̀ m e}$ bien non durable s'écrit :

$$
q_{i}=\frac{\partial \mathrm{F}(.)}{\partial c(.)} \frac{\partial c(.)}{\partial p_{i}} .
$$

On peut donc agréger la demande des $q_{i}$ pour obtenir la dépense totale en biens non durables grâce au lemme de Shephard et à la propriété d'homogénéité du premier ordre en $\mathbf{p}$ de la fonction agrégatrice $c($.$) :$

$$
y=\sum_{i} p_{i} q_{i}=\frac{\partial \mathrm{F}(\cdot)}{\partial c(.)} \sum_{i} p_{i} \frac{\partial c(.)}{\partial p_{i}}=\frac{\partial \mathrm{F}(.)}{\partial c(.)} c(.) .
$$

b. Dans une seconde étape, le ménage choisit la part de la dépense pour chaque bien dans chaque groupe (durable, non durable) selon le vecteur de prix propre à ce groupe et le niveau de l'utilité totale.

La part budgétaire de la dépense non durable $w_{i}$ dans la dépense totale $y$ devient donc :

$$
w_{i}=\frac{p_{i} q_{i}}{y}=\frac{p_{i} \frac{\partial \mathrm{F}(.)}{\partial c(.)} \frac{\partial c(.)}{\partial p_{i}}}{\frac{\partial \mathrm{F}(.)}{\partial c(.)} c(.)}=\frac{p_{i} \frac{\partial c(.)}{\partial p_{i}}}{c(.)}=\frac{p_{i}}{\partial p_{i}} \frac{\partial c(.)}{c(.)}=\frac{\partial \ln c(.)}{\partial \ln p_{i}} .
$$

Les fonctions de coût $c($.$) et d($.$) sont spécifiées comme des coûts Pig-log$ (Banks et al. [1997]) et l'équation (3) devient un système de demande Presque Idéal Quadratique (Quaids).

\section{Limites du modèle}

L'estimation des courbes d'Engel relative à toutes les consommations permet de repérer la différence entre le revenu déclaré par le ménage et celui qui correspond, selon les paramètres du modèle de demande, aux dépenses repérées dans l'enquête. On suppose souvent que les salaires servent principalement à l'achat des biens et services indispensables, alors que les revenus tirés des entreprises individuelles sont plutôt utilisés pour l'acquisition de biens durables, éventuellement de biens de luxe ${ }^{3}$. Fortin et al. [2009] ont montré récemment que les biens non durables peuvent souvent être classés comme des biens de luxe. On supposera donc ici que les revenus non salariaux peuvent être consacrés à l'achat de biens de tous types, durables ou pas.

La négligence des biens durables peut amener à sous-estimer l'estimation de la taille des marchés informels. L'examen de ces marchés en Turquie est donc particulièrement utile : selon l'Institut des statistiques de Turquie (TUIK), la

3. Lyssiotou et al. [2004] et Fortin et al. [2009]. 
part des loyers et des charges de l'habitation est de 25,9\%, alors que celle de l'alimentation s'élève à $24,9 \%$. L'importance de ces deux dépenses dans le budget des ménages montre que le biais du report des revenus sera beaucoup mieux estimé si l'on prend en compte l'ensemble des dépenses dans un système de demande.

Par ailleurs, nos estimations porteront sur les dépenses complètes des ménages (full expenditures), c'est-à-dire sur la somme des dépenses monétaire et d'une évaluation monétaire de la production domestique effectuée à partir des temps d'activité repérés dans des enquêtes de Budgets-Temps. La modélisation de cette production domestique et de la dépense complète s'opère dans le cadre d'un modèle beckerien d'allocation du temps qui est développé dans Gardes [2014].

\section{Extension du modèle de demande aux dépenses monétaires et temporelles}

Becker [1965] considère des biens finaux dont les quantités $Z_{i}, i=1$ à $m$, sont les arguments de l'utilité directe $u\left(Z_{1} Z_{2}, \ldots Z_{m}\right)$. On suppose pour simplifier que la production du bien final $i$ (par l'activité de même indice) utilise une quantité $x_{i}$ d'un panier de biens marchands de prix unitaire $p_{i}$ et un temps $t_{i}$ proportionnel à la quantité du bien marchand agrégé :

Le bien final est produit au travers d'une fonction de production domestique : $\mathrm{Z}_{i}=f_{i}\left(x_{i}, \tau_{i} ; \mathrm{W}\right)$ dépendant des caractéristiques socioéconomiques du ménage $W$. Le programme d'optimisation du ménage s'écrit donc : $\operatorname{Max} u\left(\mathrm{Z}_{1}, \mathrm{Z}_{2}, \ldots \mathrm{Z}_{m}\right)$, tel que $\mathrm{Z}_{i}=f_{i}\left(x_{i}, \tau_{i} ; \mathrm{W}\right), \sum_{i} p_{i} x_{i}=y$ et $\sum_{i} \tau_{i} x_{i}+t_{w}=\mathrm{T}$

avec $y=w t_{w}+\mathrm{V}$ le revenu monétaire du ménage (revenu du travail marchand plus autres revenus monétaires).

La considération des trois contraintes de ce programme d'optimisation permet d'écrire les prix complets du bien marchand $\tau_{i}=p_{i} x_{i}+\omega t_{i}$ et la contrainte de revenu complet en supposant que les activités domestiques dépendent d'un coût d'opportunité du temps $\omega$ qui diffère du taux de salaire net d'impôt $w$ :

$$
\sum_{i}\left(p_{i} x_{i}+\omega t_{i}\right)=y^{f}+(\omega-w)\left(\mathrm{T}-t_{w}\right)=y^{f}+(\omega-w) \sum_{i} \tau_{i} x_{i}
$$

Le revenu complet est donc corrigé, par rapport à la version beckerienne, par une fonction du temps d'activité domestique et de la différence entre le coût d'opportunité du temps des activités marchandes et non marchandes.

\section{SPÉCIFICATION ÉCONOMÉTRIQUE}

Nous supposons des prix de base unitaires : $\mathbf{p}=\mathbf{r}=1$ pour chaque ménage $h$. La courbe d'Engel s'écrit sous forme de Working issue de la minimisation de la fonction de coût Pig-Log :

$$
w_{i h}=\alpha_{i}+\beta_{i}\left[\ln \mathrm{Y}_{h}^{*}\right]+\delta_{i}\left[\ln \mathrm{Y}_{h}^{*}\right]^{2}
$$

en fonction des revenus $\mathrm{Y}_{h}$ et des paramètres $\alpha, \beta, \delta$. 
Le revenu $\mathrm{Y}_{h}^{*}$ se décompose en trois types de revenu $a, s, r:$ autres revenus, salaires, revenu d'entreprises individuelles. Le revenu déclaré est une somme pondérée de ces trois revenus :

$$
\mathrm{Y}_{h}^{*}=\sum_{m=a, s, r} \theta_{m} \mathrm{Y}_{m h} .
$$

Les poids $\left(\theta_{a}, \theta_{s}, \theta_{r}\right)$ sont supposés supérieurs à 1 en cas de report sousévalué du revenu correspondant, ou unitaires pour un juste report. On peut ainsi estimer ces pondérations au travers du système de demande, et donc la part de sous-estimation des revenus salariaux et d'entreprises individuelles. Cette estimation permet donc d'évaluer, en fonction de $\theta_{r}$ et $\theta_{s}$ (auxquels on imposera, dans l'estimation, la contrainte $\theta_{r, s} \geqslant 1$ en adoptant la spécification de Fortin et al. [2009] $\theta=\left(1+e^{k}\right)$ où $k$ devient un paramètre additionnel du modèle), la taille des revenus tirés d'occupation sur les marchés informels. On suppose donc que la vraie valeur des revenus issus d'entreprises individuelles ou des revenus d'origine salariale $\left(\mathrm{Y}_{r}^{*}\right.$ et $\left.\mathrm{Y}_{s}^{*}\right)$ s'écrivent respectivement : $\left(1+e^{k}\right)^{*} \mathrm{Y}_{r}$ et $\left(1+e^{v}\right)^{*} \mathrm{Y}_{s}$ où $v$ représente le paramètre de biais de report pour les salariés ${ }^{4}$.

On peut finalement déterminer la somme des proportions des sous-reports par rapport au revenu total : $y_{\mathrm{m}}=\mathrm{Y}_{m} / \mathrm{Y}$, où $\mathrm{Y}$ est la somme de tous les revenus (incluant les transferts sociaux). La fonction (5) peut donc être écrite grâce aux équations (6) :

$$
\begin{aligned}
w_{i h} & =\alpha_{i}+\sum_{j} \alpha_{i j} z_{j h}+\sum_{n=1}^{3} \lambda_{i n}\left(y_{r, s}\right)^{2}+\left[\ln \mathrm{Y}_{h}+\ln \left(\sum_{m=a, s, r} \theta_{m} y_{m}\right)\right] \\
& +\delta_{i}\left[\ln \mathrm{Y}_{h}+\ln \left(\sum_{m=a, s, r} \theta_{m} y_{m}\right)\right]^{2}+e_{i h}
\end{aligned}
$$

où $\mathrm{Z}$ sont les caractéristiques des ménages qui permettent de représenter l'hétérogénéité des préférences. Nous introduisons, comme Lyssiotou et al. [2004] et Fortin et al. [2009], le terme $\sum_{n=1}^{3} \lambda_{i n}\left(y_{r, s}\right)^{n}$ dans chaque équation afin de tenir compte du poids relatif différencié des revenus salariaux ou d'entrepreneurs individuels selon le type de ménage. Cet ajout tend à réduire la confusion possible dans l'estimation entre hétérogénéité des réactions des consommateurs et sous-estimation des reports de revenus.

\section{L'APPARIEMENT DES DONNÉES MICROÉCONOMIQUES}

Nous utilisons deux enquêtes auprès de ménages effectuées par TUIK ${ }^{5}$ : Budget de Famille (BdF) et Budget de Temps (BdT).

En 2003, l'enquête BdF a été réalisée auprès de 2160 ménages chaque mois, soit un total de 25920 ménages dans l'année. En 2004, 2005 et 2006, le nombre

4. L'estimation est effectuée en deux étapes : l'équation (7) fournit d'abord le paramètre de sous-report des revenus d'entreprises individuelles et le revenu déclaré par ce type de travailleur est multiplié par ce paramètre $\theta_{r}$. En deuxième étape, le biais de sous-report des salaires est estimé par la même équation (7). On trouvera le détail des revenus ajustés des entrepreneurs individuels en Annexe II.

5. Institut des statistiques de Turquie. 
de ménages enquêtés a été de 720 par mois et de 8640 dans l'année. Trois groupes de variables ont été sélectionnés à partir de ces données :

1. Les variables du statut socioéconomique des ménages (type d'habitation, statut d'occupation, système de chauffage, confort de l'habitation, moyens de transport, etc.) ;

2. Les variables liées aux individus sont : l'âge, le sexe, le niveau d'éducation et les variables liées à l'emploi (profession, type d'activité économique, performance au travail). L'intérêt majeur des données BdF est l'ensemble des 72 variables de revenus ${ }^{6}$;

3. Les variables de consommation (l'alimentation, les boissons non alcoolisées, les boissons alcoolisées, le tabac et cigarettes, l'habillement, la santé, le transport, l'éducation, les services, etc.).

Réalisée en 2006, l'enquête Budget de Temps (BdT) a interrogé environ 390 ménages chaque mois, ce qui donne un total de 5070 ménages dans l'année. Dans ces ménages, 11815 personnes âgées de plus de 15 ans ont été interrogées et on leur a demandé de remplir deux carnets : un pour un jour de la semaine et un pour un jour de week-end. Les personnes enquêtées remplissaient leurs activités journalières sur vingt-quatre heures avec les intervalles de dix minutes. Cette enquête BdT de 2006 est appariée indépendamment avec les quatre vagues de l'enquête $\mathrm{BdF}$, ce qui donne une série temporelle des données transversales contenant à la fois des informations sur les dépenses monétaires et les dépenses de temps ${ }^{7}$.

\section{La méthode d'appariement}

Nous réunissons les dépenses monétaires et les dépenses de temps dans une activité économique unique au niveau du ménage. L'appariement est effectué par une régression sur les Budgets-Temps. Cet ajustement sert à prédire le temps de chaque activité pour chaque ménage du BdF en utilisant les mêmes variables dans les deux sources telles que l'âge du chef de famille, le statut matrimonial, la possession d'un téléphone portable, le nombre de personnes dans le ménage ou la localisation géographique. La prédiction est effectuée séparément pour le chef du ménage et son conjoint ${ }^{8}$.

Plus précisément, nous déterminons huit types d'activités dans le BdT qui sont compatibles avec les données disponibles dans le BdF :

- le temps de préparation des repas $(\mathrm{BdT})$ - les dépenses d'alimentation $(\mathrm{BdF})$;

- le temps consacré aux soins personnels et à la santé $(\mathrm{BdT})$ - les dépenses de soins personnels et de santé $(\mathrm{BdF})$;

6. Dans l'approche monétaire, nous éliminons tous les ménages déclarant un revenu négatif. Toutefois, dans l'approche des dépenses complètes, nous n'avons pas besoin d'éliminer les ménages dont les revenus non salariaux ou les revenus de salariés obtenus sur le marché formel sont supérieurs au total des revenus déclarés.

7. Nous ne tenons pas compte dans les estimations d'éventuelles corrélations spatiales entre régions.

8. Sont pris en compte uniquement les ménages avec les valeurs positives du temps passé sur leurs activités. 
- le temps de ménage et d'entretien de la maison $(\mathrm{BdT})$ - les dépenses de logement $(\mathrm{BdF})$;

- le temps d'entretien des vêtements $(B d T)$ - les dépenses d'habillement $(\mathrm{BdF})$;

- le temps consacré à l'éducation $(\mathrm{BdT})$ - les dépenses d'éducation $(\mathrm{BdF})$;

- le temps de transport $(\mathrm{BdT})$ - les dépenses de transport $(\mathrm{BdF})$;

- le temps de loisirs $(\mathrm{BdT})$ - les dépenses de loisirs $(\mathrm{BdF})$;

- le temps des activités diverses $(\mathrm{BdT})$ - les dépenses diverses $(\mathrm{BdF})$.

Le temps consacré à l'alimentation contient aussi le temps des services au sein de la famille ${ }^{9}$. Le temps de soins personnels est composé des services commerciaux, de gestion, d'aide aux malades ou aux personnes âgées. Le temps consacré au ménage et à l'entretien de la maison comprend également les activités de jardinage, les soins pour les animaux domestiques, les réparations, la rénovation et la gestion. Le temps d'entretien des vêtements contient aussi le lavage et le repassage des vêtements. Dans le temps consacré à l'éducation sont incluses des activités d'apprentissage, d'étude et aussi la garde des enfants. Le temps de transport contient essentiellement le temps consacré aux voyages et autres activités non spécifiées. Le temps de loisirs représente des activités du travail bénévole, la participation à des réunions, la vie sociale et les divertissements, les activités sportives, la chasse, la pêche, les hobbys et jeux, l'écoute de musique, le visionnage de films ou de vidéos, etc. Le temps des activités diverses contient en particulier le temps de la recherche d'emploi.

\section{Valorisation du temps}

Compte tenu du fait que les individus utilisent leur temps pour produire ces biens et services et que ce temps a un coût, nous considérons le revenu complet du ménage comme la somme de son revenu monétaire et du coût du temps. Nous utilisons deux méthodes de valorisation de temps passé en activité domestique. La première méthode consiste à imputer un salaire potentiel net des impôts et des prélèvements aux individus sans emploi par la méthode de sélection d'Heckman à deux étapes en supposant que l'utilisation du temps est parfaitement échangeable entre les activités marchandes et non marchandes. Le logarithme du revenu mensuel, l'âge du chef de famille, l'âge au carré, le nombre d'enfants, le nombre de personnes au sein du ménage sont utilisés pour prédire le taux de salaire sousjacent des personnes qui ne travaillent pas ${ }^{10}$. Le coût d'opportunité du travail non marchand pour l'homme et la femme qui ne travaillent pas est donc estimé comme le taux de salaire espéré sur le marché de travail. La deuxième méthode de valorisation du temps consiste à appliquer le salaire minimum officiel en Turquie en $2006^{11}$.

9. Le temps de l'alimentation concerne seulement la préparation des repas d'un fait de l'impossibilité de séparer l'activité de prise de repas des activités de soins personnels.

10. Les résultats d'estimation du modèle de sélection à deux étapes d'Heckman se trouvent dans le tableau A3 de l'Annexe I.

11. On peut noter que le coût d'opportunité se situe plutôt entre ces deux valeurs. Pour plus de détails, voir Gardes [2014]. 


\section{RÉSULTATS EMPIRIQUES}

Nous estimons le système complet de demande (équation 7) en utilisant la méthode des moments généralisés (MMG) à la fois pour la dépense complète (temps plus argent) et pour la seule dépense monétaire. On peut noter que les prix ne sont pas inclus dans l'équation (cette estimation sera opérée ultérieurement). La variable de revenu étant supposée endogène, nous avons utilisé les instruments suivants : l'âge du chef du ménage et de son conjoint, le statut matrimonial du ménage, le nombre d'enfants âgés de plus de 16 ans, la possession de logement, l'emploi en CDI pour le chef de ménage, le type d'occupation du chef de ménage, le niveau d'éducation du chef de ménage, la possession de biens durables (télévision, Internet, réfrigérateur, congélateur, lave-vaisselle, four).

Les variables de contrôle suivantes ont été incluses dans le modèle : le nombre de personnes au sein du ménage, le nombre de pièces dans le logement, le statut du logement, le nombre de personnes de moins de 16 ans, la localisation (urbaine, rurale), le fait que le conjoint masculin travaille comme ouvrier ou qu'il travaille comme cadre, le fait que conjoint féminin travaille comme ouvrière ou comme cadre, qu'elle travaille comme ouvrière dans une entreprise de moins de dix personnes ${ }^{12}$, que le conjoint masculin n'ait pas de contrat de travail, que le conjoint féminin n'ait pas de contrat de travail et les variables muettes pour la présence des biens durables tels que l'ordinateur, la voiture ou un système de chauffage.

Les résultats de l'estimation du modèle pour la dépense complète, ainsi que ceux correspondant à la seule dépense monétaire, sur les données empilées des enquêtes couvrant la période 2003-2006, sont présentés respectivement dans le tableau 3 et le tableau A2 dans l'annexe ${ }^{13}$. Le nombre d'observations dans l'échantillon empilé est de 34414 ménages. Sont présentés uniquement les paramètres estimés de sept (sur huit) équations de coefficients budgétaires, la huitième (autres biens et services) étant redondante à cause de la condition d'additivité. Le test d'instrumentation de la variable revenu indique que les instruments utilisés ne sont pas faibles : le test The StockYogo Test avec la méthode de biais rejette l'hypothèse de présence des instruments faibles ${ }^{14}$; la statistique $\mathrm{F}$ - de la première étape $(19,80)$ est supérieure à la valeur critique pour le biais maximal de $10 \%$ de la variable instrumentale $(11,49)$.

Nous calculons la taille de l'économie informelle pour différents types de revenus (revenus non salariaux et revenus salariaux) à la fois dans la version avec les dépenses complètes (temps + argent) et dans celle concernant les seules dépenses monétaires (argent exclusivement). L'évaluation est obtenue par calibrage des paramètres de sous-déclaration $k$ et $v$ avec

12. Cette variable est omise dans l'estimation de la dépense complète.

13. Dans l'estimation basée sur les variables de l'année 2003, la restriction de sur-identification est de 25,84 avec 25 degrés de liberté. La valeur $p$ de Chi-carré pour l'estimation sur les données monétaires est de 0,41 supérieure à 0,05 et 1 'hypothèse de validité des instruments ne peut donc pas être rejetée pour l'ensemble des variables de contrôle présentes. Nous gardons les mêmes variables de contrôle sans en ajouter d'autres dans les deux estimations sur les dépenses complètes et monétaires.

14. Les résultats d'estimation se trouvent dans le tableau 3. 
la part des revenus non salariaux et salariaux dans le PIB ( $c f$. respectivement le tableau A4 et le tableau A5). Les résultats sont présentés dans le tableau 1 .

La part de l'économie informelle dans le PIB, estimée avec les dépenses monétaires uniquement et basée sur les revenus non salariaux, diminue pour la période (2003-2006) de 33,99 à 28,9\%. L'approche par la dépense complète (temps + argent) donne des parts d'informel plus élevées de 41,22 à 35,1\% de PIB pour la même période. La sous-déclaration des revenus salariaux augmente de 13 à $15 \%$ de PIB entre 2003 et 2006 avec l'approche monétaire et de 19 à $22 \%$ avec l'approche de la dépense complète.

La taille totale de l'économie informelle est la somme de deux sources (revenus salariaux et revenus non salariaux) et elle diminue de $47 \%$ de PIB en 2003 à $44 \%$ de PIB en 2006 en considérant les seules dépenses monétaires. Les chiffres correspondants pour l'approche en termes de dépenses complètes donnent une évolution de la taille totale de l'informel de 60 à $57 \%$ de PIB pour la même période 2003-2006.

Tableau 1. La taille de l'économie informelle entre 2003 et 2006

(\% du PIB)

\begin{tabular}{l|c|c|c|c|c}
\hline \multicolumn{1}{c|}{ Année } & 2003 & 2004 & 2005 & 2006 & Moyenne \\
\hline $\begin{array}{l}\text { Taille de l'économie informelle pour l'estimation } \\
\text { des dépenses monétaires (TI) }\end{array}$ & 33,99 & 35,98 & 35,00 & 28,90 & 33,46 \\
$\begin{array}{l}\text { Taille de l'économie informelle pour l'estimation } \\
\text { des dépenses monétaires (TS) }\end{array}$ & 12,94 & 13,91 & 14,55 & 15,03 & 14,11 \\
$\begin{array}{l}\text { Taille de l'économie informelle pour l'estimation } \\
\text { des dépenses monétaires (TI + TS) }\end{array}$ & 46,93 & 49,89 & 49,55 & 43,93 & 47,57 \\
\hline $\begin{array}{l}\text { Taille de l'économie informelle pour l'estimation } \\
\text { des dépenses complètes (TI) }\end{array}$ & 41,22 & 43,64 & 42,50 & 35,10 & 40,61 \\
$\begin{array}{l}\text { Taille de l'économie informelle pour l'estimation } \\
\text { des dépenses complètes (TS) }\end{array}$ & 19,01 & 20,43 & 21,37 & 22,08 & 20,72 \\
$\begin{array}{l}\text { Taille de l'économie informelle pour l'estimation } \\
\text { des dépenses complètes (TI + TS) }\end{array}$ & 60,23 & 64,07 & 63,87 & 57,18 & 61,30 \\
\hline
\end{tabular}

La part non déclarée des revenus salariaux peut être utilisée pour évaluer les gains des employeurs provenant de la sous-déclaration des contributions sociales et fiscales sur les salaires en Turquie. Les enquêtes utilisées permettent d'obtenir le taux des cotisations assises sur les salaires (entre 21,5 et $27 \%$ selon le secteur de l'économie). On obtient donc la part non déclarée des contributions sociales et fiscales assises sur les salaires d'ouvriers en multipliant ces taux de cotisations par les taux de l'économie informelle obtenue pour les revenus salariaux et ceci pour chaque année de la période considérée. La part des contributions non payées par les employeurs augmente de 3,1\% de PIB en 2003 à 3,7 de PIB en 2006 (cf. tableau 2). 
Tableau 2. Les cotisations sociales impayées de l'employeur entre 2003 et 2006

(\% du PIB)

\begin{tabular}{c|c|c|c|c|c}
\hline Année & 2003 & 2004 & 2005 & 2006 & Moyenne \\
\hline Cotisations sociales impayées de l'employeur & $2,78-3,49$ & $3,00-3,75$ & $3,12-3,92$ & $3,23-4,06$ & $3,03-3,81$ \\
\hline
\end{tabular}

La prise en compte des activités domestiques (par l'estimation sur dépenses complètes) conduit à une augmentation significative du ratio de la sous-déclaration et, par conséquent, de l'évaluation de la taille du secteur informel. $(+21 \%$ et $+47 \%$ ) respectivement pour les indépendants et les salariés. L'évolution de la taille de l'informel en 2006 s'explique par la diminution de la part des revenus non salariaux et par l'augmentation de la part des revenus salariaux dans le PIB (respectivement de 14,4\% et 16,1\%; $c f$. tableau A5 dans l'Annexe I).

Quelle pourrait être la raison de la baisse de la taille de l'informel après 2004 ? On peut constater, à partir de la statistique macro de TUIK, que la différence entre ces années peut provenir à la fois de la baisse de l'inflation et de la hausse du revenu disponible en 2004. L'indice de prix à la consommation (CPI) augmente de $9,3 \%$ et de $7,7 \%$ respectivement en 2004 et en 2005, tandis qu'il augmente de $29,7 \%$ et de $18,4 \%$ respectivement en 2002 et en 2003. Selon les chiffres annuels de TUIK sur l'emploi et le revenu disponible moyen, le revenu disponible provenant des activités des indépendants a augmenté de 22,5\% en 2004 par rapport à 2003 et a continué à augmenter dans les années suivantes ${ }^{15}$. L'indice de salaire réel d'ouvrier indique également que le salaire réel a baissé dans la période considérée pour les ouvriers du secteur, public privé et pour le salaire minimum et a augmenté uniquement pour les fonctionnaires $(-2,7 \%,-0,7 \%$, $-0,9 \%$ et $+6,2 \%$ respectivement). De plus le taux de participation sur le marché de travail et le taux de chômage ont augmenté à partir de 2004. En revanche, le sous-emploi a diminué entre 2004 et $2006^{16}$. Ces évolutions économiques exceptionnelles pourraient expliquer la diminution progressive de nos estimations de la taille de l'informel après 2004, particulièrement celles obtenues par l'approche de la dépense complète (temps + argent).

Notre estimation de la taille de l'économie informelle par l'approche monétaire et uniquement pour les revenus non salariaux est proche de celle obtenue par d'autres auteurs utilisant les méthodes macroéconomiques (par exemple, Schneider et Sava an [2007] avec le modèle DYMIMIC), tandis que l'utilisation du concept de dépense complète donne des résultats significativement supérieurs. On peut noter que notre évaluation en termes de dépense monétaire donne une taille de l'informel en 2005 de l'ordre de $48 \%$ de PIB, soit 27 points de pourcentage de plus que les résultats sur la base des données microéconomiques de Davutyan [2008] (21\%; $f$. tableau A6). La différence peut provenir du fait que Davutyan [2008] a utilisé le modèle de demande à une seule équation, celle de la dépense alimentaire. Dans notre approche, nous utilisons un modèle complet de demande qui prend en compte l'ensemble des dépenses de biens et services, ce qui permet d'obtenir les résultats d'estimation plus fiables, en particulier pour le paramètre de la sous-déclaration du revenu, par le fait qu'on tient ainsi compte des possibles substitutions entre les dépenses.

15. Cette croissance a été de 5,94\% en 2005 par rapport à 2004 .

16. Les taux de sous-emploi entre 2004-2006 étaient de 10,8\%; 10,6 \%; 10,2\%. 
焉

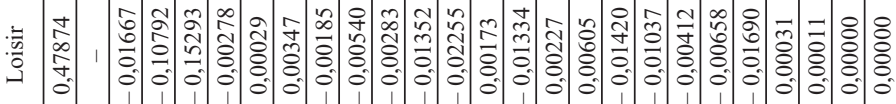

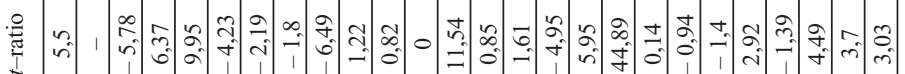

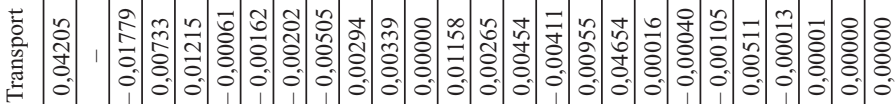

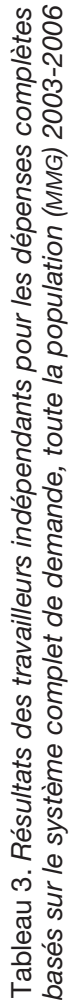

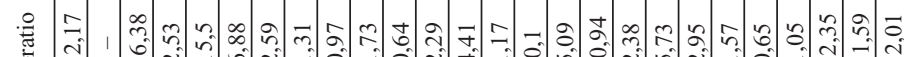

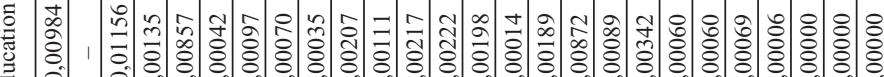

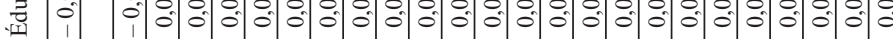

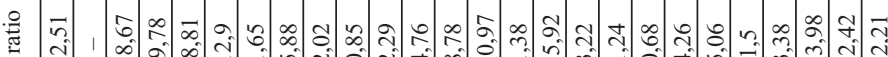

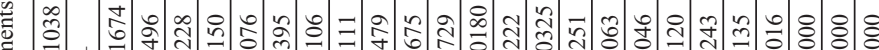

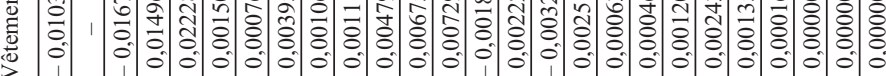

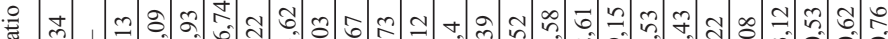

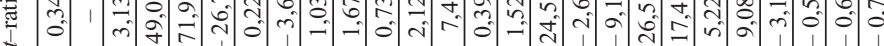

苛

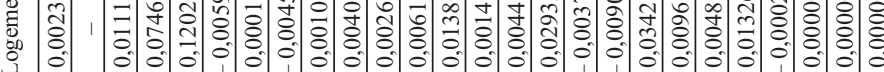

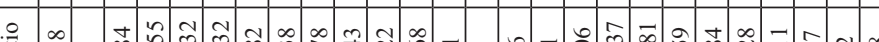

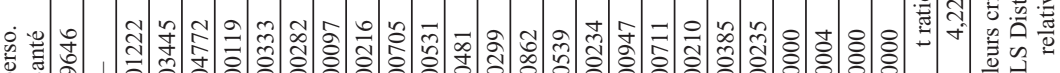

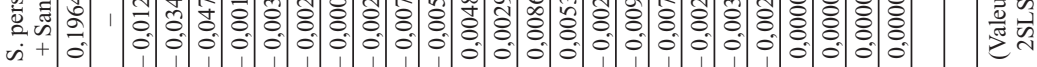

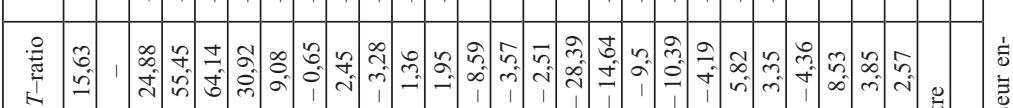

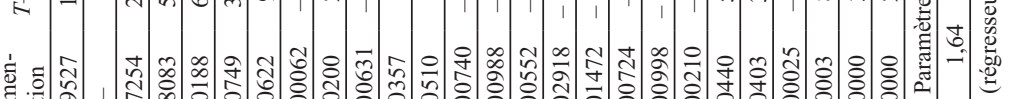

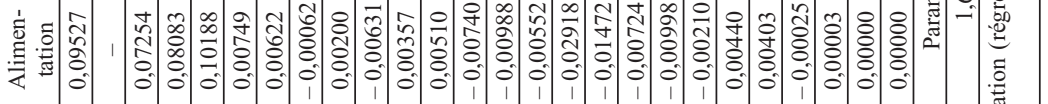

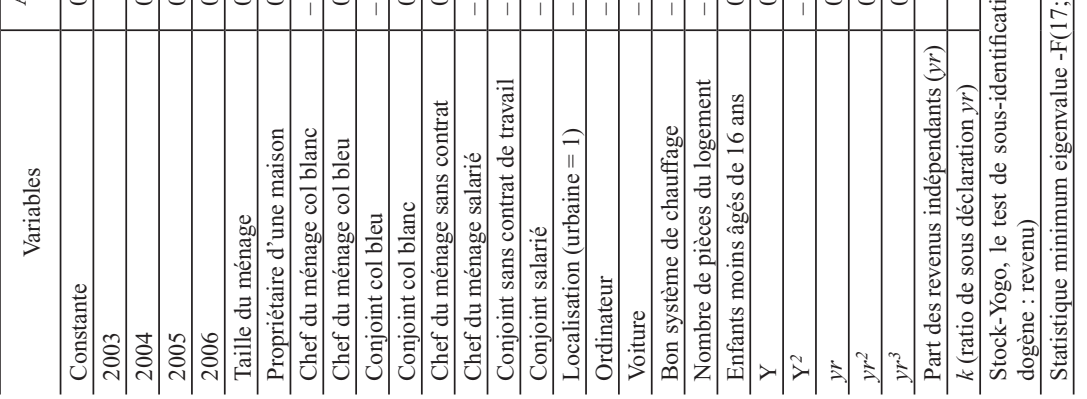




\section{CONCLUSION}

Nous utilisons une nouvelle méthode pour estimer la part sous-déclarée du revenu des ménages avec des données microéconomiques sur le budget monétaire des ménages et sur leur budget de temps. Deux approches sont présentées et comparées : une plutôt conventionnelle qui s'appuie sur les mesures exclusivement monétaires et l'autre, originale, qui intègre les dépenses monétaires et les dépenses du temps dans le concept beckerien de la dépense complète (full expenditure). Cette unification des dépenses monétaires et de temps a été obtenue par un appariement statistique de l'enquête classique de budget des familles et de l'enquête sur les budgets de temps. C'est la première fois que cette méthode est appliquée dans un pays en développement - la Turquie (les applications antérieures concernaient la Grande-Bretagne et le Québec pour les seules dépenses monétaires). Les résultats montrent la grande importance des activités domestiques dans l'estimation de la taille de l'économie informelle en Turquie.

Le modèle est bien estimé avec presque tous les paramètres significatifs. Nous considérons tous les biens et services en tenant compte de la production domestique dans le cadre d'un système de demande en additionnant la valorisation monétaire du temps passé aux différentes activités avec les dépenses monétaires correspondantes.

La taille de l'économie informelle estimée par cette méthode de la dépense complète, pour la période 2003-2006 en Turquie, s'élève à $61 \%$ du PIB à comparer avec $48 \%$ obtenus par la méthode conventionnelle utilisant exclusivement les dépenses monétaires. Dans les deux estimations, nous considérons à la fois la sous-déclaration des revenus non salariaux et des revenus salariaux.

Quand on compare les résultats obtenus pour la Turquie avec ceux du Québec (Fortin et al. [2009] qui s'appuient sur une méthodologie identique mais estiment sur les seules dépenses monétaires), on observe une différence très grande dans la taille de l'informel : au Québec, l'estimation (sur données monétaires) de l'économie informelle représente $6 \%$ de PIB en 2002, alors qu'elle atteint 46,9\% en Turquie en 2003 (dans le même cadre de l'approche monétaire).

Notre approche originale intégrant la dépense complète et le revenu complet renforce la robustesse des résultats d'estimation par rapport à des applications précédentes. De plus, le concept de dépenses et de revenu complets donne la possibilité de calculer des prix individuels (complets) et le coût d'opportunité du temps pour chaque ménage, ce qui permettra une estimation plus adéquate du système de demande avec toutes les contraintes théoriques (voir Gardes [2014]).

\section{RÉFÉRENCES BIBLIOGRAPHIQUES}

AKalin G., KesIKOgLu F. [2007], « Turkiye'de Kayitdisi Ekonomi ve Buyume Iliskisi » (La relation entre l'économie souterraine et la croissance économique en Turquie), Uluslararası Yönetim İktisat ve İsletme Dergisi, 3 (5), p. 71-87.

BALDEMIR E., OZKoc H., IsCi O. [2009], « MIMIC Model ve Yolsuzluk Uzerine Turkiye Uygulamasi » (Le modèle MIMIC et l'application sur la corruption en Turquie), Dokuz Eylul Universitesi Iktisadi ve Idari Bilimler Fakultesi, 24 (2), p. 49-63. 
BANKs J., Blundell R., Lewbel A. [1997], « Quadratic Engel Curves and Consumer Demand », Review of Economic Studies, 89 (4), p. 527-539.

BECKER G. [1965], "A theory of the allocation of time », The Economic Journal, 75, p. $493-517$

Cetintas H., Vergil H. [2003], « Türkiye'de Kayıtdışı Ekonominin Tahmini » (Estimation de l'économie souterraine en Turquie), Doğuş Üniversitesi Dergisi, 4 (1), p. 15-30.

DAVUTYAN N. [2008], « Estimating the Size of Turkey's Informal Sector: An Expenditure Based Approach », ERF Working Paper, 403.

FEINSTEIN J. [1999], « Approaches for estimating noncompliance: examples from 21 Federal taxation in the United States », Economic Journal, 109, p. 360-369.

Fortin B., LACroIX G., PINARD D. [2009], « Évaluation de l'économie souterraine au Québec : une approche micro-économétrique », Revue économique, 60 (5), p. $1257-1274$.

GARDES F. [2014], «Full Price Elasticities and the Opportunity Cost for Time», document de travail, Centre d'économie de la Sorbonne, Université Paris I Panthéon-Sorbonne.

ILGIN Y. [2002], « Kayıtdışı Ekonomiyi Tahmin Yöntemleri ve Türkiye'de Durum » (Les méthodes d'estimation de l'économie informelle et le cas de la Turquie), Planlama Dergisi, DPT'nin Kuruluşunun 42, Y11ı Özel Say1, p. 145-156.

ILKKARACAN A.I., UMUT G. [2009], « Time-use, the Value of Non-Market Production and its Interactions with the Market Sector: The Case of Turkey », Paper presented at International Conference on Inequalities and Development in the Mediterranean Countries, Mimeo.

Institut DeS STATISTIQUES DE TURQUie [2006, 2005, 2004, 2003], Enquêtes Budget de Famille.

Institut DES STATISTIQUeS DE TURQUie [2006], Enquête Emploi du Temps.

Kasnakoglu Z., Dayioglu M. [2002], « Measuring the Value of Home Production in Turkey » dans BulutaY T. (eds), New Developments in National Accounts (In the context of strengthening the capacity in national accounts project implemented together with UNDP and SIS the new developments in national accounts), Ankara, Republic of Turkey Prime Ministry State Institute of Statistics p. 73-97.

Lyssiotou P., PAshardes P., Stengos T. [2004], « Estimates of the Black Economy based on Consumer Demand Approaches », The Economic Journal, 114, p. 622-640.

OGunC F., YilmaZ G. [2000], « Estimating Underground Economy in Turkey », Discussion Paper, Central Bank-Republic of Turkey.

OzSOYLu A.F. [1996], Türkiye'de Kayıtdışı Ekonomi (Économie informelle en Turquie), Istanbul, Baglam.

PISSARIDES C., WeBer G. [1989], « An Expenditure-Based Estimate of Britain’s Black Economy », Journal of Public Economics, 39, p. 17-32.

SCHNEIDER F., ENSTE D. [2000], « Shadow Economies: size, causes and consequences », Journal of Economic Literature, 38, p. 77-114.

SCHNEIDER F., SAVAŞAN F. [2007], « Dymimic Estimates of the Size of Shadow Economies of Turkey and of Her Neighbouring Countries ", International Research Journal of Finance and Economics, 9, p. 126-143.

Stock J.H., Wright J.H., Yogo M. [2002], « A Survey of Weak Instruments and Weak Identification in Generalized Method of Moments ", Journal of Business and Economic Statistics, 20, p. 518-529.

THOMAS J. [1999], « Quantifying the black economy: Measurement without theory yet again? », Economic Journal, 109 (456), p. 381-389.

Ulgen S., OzTURK U. [2006], Kayitdisi Ekonomi ve Surdurulebilir Buyume: AB yolunda Degerlendirme ve Cozum Onerileri (L'économie informelle et la croissance durable : propositions de solutions sur la route de l'UE), TUSIAD Buyume Stratejileri Dizisi N 8, $\mathrm{T} / 2007-01 / 428$.

Us V. [2004], « Kayitdisi Ekonomi Tahmini Yontem Onerisi: Turkiye Ornegi » (Une proposition pour la méthode d'estimation de l'économie informelle : le cas de la Turquie), Turkiye Ekonomi Kurumu, 2004/17. 


\section{ANNEXES}

ANNEXE I

Tableau A1. Statistiques descriptives

\begin{tabular}{|c|c|c|c|c|c|c|}
\hline Part du budget & Variables & $\mathrm{N}$ & Moyenne & Écart type & Minimum & Maximum \\
\hline \multirow{7}{*}{$\begin{array}{l}\text { DÉPENSES } \\
\text { MONÉTAIRES }\end{array}$} & Alimentation & 34414 & 0,3139 & 0,1528 & 0 & 1 \\
\hline & Soins personnels + Santé & 34414 & 0,0782 & 0,0756 & 0 & 0,8362 \\
\hline & Logement & 34414 & 0,3336 & 0,1398 & 0 & 1 \\
\hline & Vêtements & 34414 & 0,0586 & 0,0703 & 0 & 0,5893 \\
\hline & Éducation & 34414 & 0,0117 & 0,0465 & 0 & 0,8323 \\
\hline & Transport & 34414 & 0,0799 & 0,0982 & 0 & 0,8723 \\
\hline & Loisir & 34414 & 0,0586 & 0,057 & 0 & 0,8859 \\
\hline Part du budget & Variables & $\mathrm{N}$ & Moyenne & Écart type & Minimum & Maximum \\
\hline \multirow{7}{*}{$\begin{array}{l}\text { DÉPENSES } \\
\text { COMPLĖTES }\end{array}$} & Alimentation & 34414 & 0,16 & 0,0744 & 0,0154 & 0,7459 \\
\hline & Soins personnels + Santé & 34414 & 0,1441 & 0,0427 & 0,0071 & 0,6846 \\
\hline & Logement & 34414 & 0,1716 & 0,0896 & 0,0261 & 0,904 \\
\hline & Vêtements & 34414 & 0,0327 & 0,0375 & 0,0004 & 0,4431 \\
\hline & Éducation & 34414 & 0,0097 & 0,0282 & 0,0001 & 0,7469 \\
\hline & Transport & 34414 & 0,0825 & 0,0619 & 0,007 & 0,7838 \\
\hline & Loisir & 34414 & 0,2678 & 0,0796 & 0,0177 & 0,8674 \\
\hline \multirow[t]{5}{*}{ Part du revenu } & Variables & $\mathrm{N}$ & Moyenne & Écart type & Minimum & Maximum \\
\hline & $\begin{array}{l}\text { Travailleurs indépendants } \\
\text { / Revenu total }\end{array}$ & 34414 & 61,701 & 387,1789 & 0 & 20000 \\
\hline & $\begin{array}{l}\text { Travailleurs salariés } \\
\text { / Revenu total }\end{array}$ & 34414 & 71,4754 & 261,7365 & 0 & 7380 \\
\hline & $\begin{array}{l}\text { D'autre revenu } \\
\text { / Revenu total* }\end{array}$ & 34414 & 106,7364 & 349,9777 & 0 & 12000 \\
\hline & $\ln ($ Revenu total $)$ & 34414 & 6,6002 & 0,872 & 0,08 & 11,0532 \\
\hline \multirow[t]{4}{*}{$\begin{array}{l}\text { Caractéristiques } \\
\text { démographiques }\end{array}$} & Variables & $\mathrm{N}$ & Moyenne & Écart type & Minimum & Maximum \\
\hline & Nombre des enfants & 34414 & 1,4072 & 1,4372 & 0 & 13 \\
\hline & $\begin{array}{l}\text { Enfants moins âgés } \\
\text { de } 16 \text { ans }\end{array}$ & 34414 & 0,644 & 0,4788 & 0 & 1 \\
\hline & Taille du ménage & 34414 & 4,3325 & 1,9661 & 1 & 23 \\
\hline \multirow[t]{4}{*}{$\begin{array}{l}\text { Catégories } \\
\text { socioprofessionnelles }\end{array}$} & Variables & $\mathrm{N}$ & Moyenne & Écart type & Minimum & Maximum \\
\hline & Chef du ménage col blanc & 34414 & 0,2075 & 0,4055 & 0 & 1 \\
\hline & Chef du ménage col bleu & 34414 & 0,3681 & 0,4823 & 0 & 1 \\
\hline & $\begin{array}{l}\text { Chef du ménage d'autre } \\
\text { profession }\end{array}$ & 34414 & 0,4241 & 0,4942 & 0 & 1 \\
\hline
\end{tabular}




\begin{tabular}{|c|c|c|c|c|c|c|}
\hline & $\begin{array}{l}\text { Chef du ménage } \\
\text { sans contrat }\end{array}$ & 34414 & 0,0314 & 0,1745 & 0 & 1 \\
\hline & $\begin{array}{l}\text { Chef du ménage } \\
\text { travaillant dans } \\
\text { une société (moins } \\
\text { de } 10 \text { travailleurs) }\end{array}$ & 34414 & 0,5379 & 0,4985 & 0 & 1 \\
\hline & Chef du ménage salarié & 34414 & 0,521 & 0,4995 & 0 & 1 \\
\hline & $\begin{array}{l}\text { Chef du ménage } \\
\text { travailleur formel }\end{array}$ & 34414 & 0,529 & 0,4991 & 0 & 1 \\
\hline & Conjoint col blanc & 34414 & 0,0298 & 0,17 & 0 & 1 \\
\hline & Conjoint col bleu & 34414 & 0,0505 & 0,2191 & 0 & 1 \\
\hline & $\begin{array}{l}\text { Conjoint d'autre } \\
\text { profession }\end{array}$ & 34273 & 0,9233 & 0,2659 & 0 & 1 \\
\hline & Conjoint sans contrat & 34414 & 0,0156 & 0,1242 & 0 & 1 \\
\hline & $\begin{array}{l}\text { Conjoint travaillant } \\
\text { dans une société } \\
\text { (moins de } 10 \text { travailleurs) }\end{array}$ & 34414 & 0,2061 & 0,4045 & 0 & 1 \\
\hline & Conjoint salarié & 34414 & 0,055 & 0,2279 & 0 & 1 \\
\hline & $\begin{array}{l}\text { Conjoint travailleur } \\
\text { formel }\end{array}$ & 34414 & 0,0522 & 0,2224 & 0 & 1 \\
\hline \multirow[t]{2}{*}{$\begin{array}{l}\text { Localisation } \\
\text { régionale }\end{array}$} & Variable & $\mathrm{N}$ & Moyenne & Écart type & Minimum & Maximum \\
\hline & Urbaine $(=1)$ & 34414 & 0,6651 & 0,4719 & 0 & 1 \\
\hline \multirow[t]{13}{*}{$\begin{array}{l}\text { Biens durables } \\
\text { et luxes }\end{array}$} & Variables & $\mathrm{N}$ & Moyenne & Écart-type & Minimum & Maximum \\
\hline & Voiture & 34414 & 0,2622 & 0,4398 & 0 & 1 \\
\hline & Télévision & 34414 & 0,9775 & 0,1481 & 0 & 1 \\
\hline & $\begin{array}{l}\text { Bon système de chauffage } \\
\text { (avec chauffage central) }\end{array}$ & 34414 & 0,1754 & 0,3803 & 0 & 1 \\
\hline & Câble TV & 34414 & 0,0373 & 0,1895 & 0 & 1 \\
\hline & Ordinateur & 34414 & 0,1213 & 0,3265 & 0 & 1 \\
\hline & Internet & 34414 & 0,0426 & 0,202 & 0 & 1 \\
\hline & Réfrigérateur & 34414 & 0,9797 & 0,1409 & 0 & 1 \\
\hline & Congélateur & 34414 & 0,0411 & 0,1986 & 0 & 1 \\
\hline & Lave-vaisselle & 34414 & 0,2219 & 0,4155 & 0 & 1 \\
\hline & Four & 34414 & 0,0496 & 0,2171 & 0 & 1 \\
\hline & Climatisation & 34414 & 0,0385 & 0,1924 & 0 & 1 \\
\hline & Téléphone portable & 34414 & 0,6761 & 0,4679 & 0 & 1 \\
\hline \multirow[t]{4}{*}{ Logement } & Variables & $\mathrm{N}$ & Moyenne & Écart type & Minimum & Maximum \\
\hline & $\begin{array}{l}\text { Propriétaires } \\
\text { de plein droit }\end{array}$ & 34414 & 0,6673 & 0,4711 & 0 & 1 \\
\hline & Accédant à la propriété & 34414 & 0,0271 & 0,1624 & 0 & 1 \\
\hline & Logement loué & 34414 & 0,2488 & 0,4323 & 0 & 1 \\
\hline
\end{tabular}




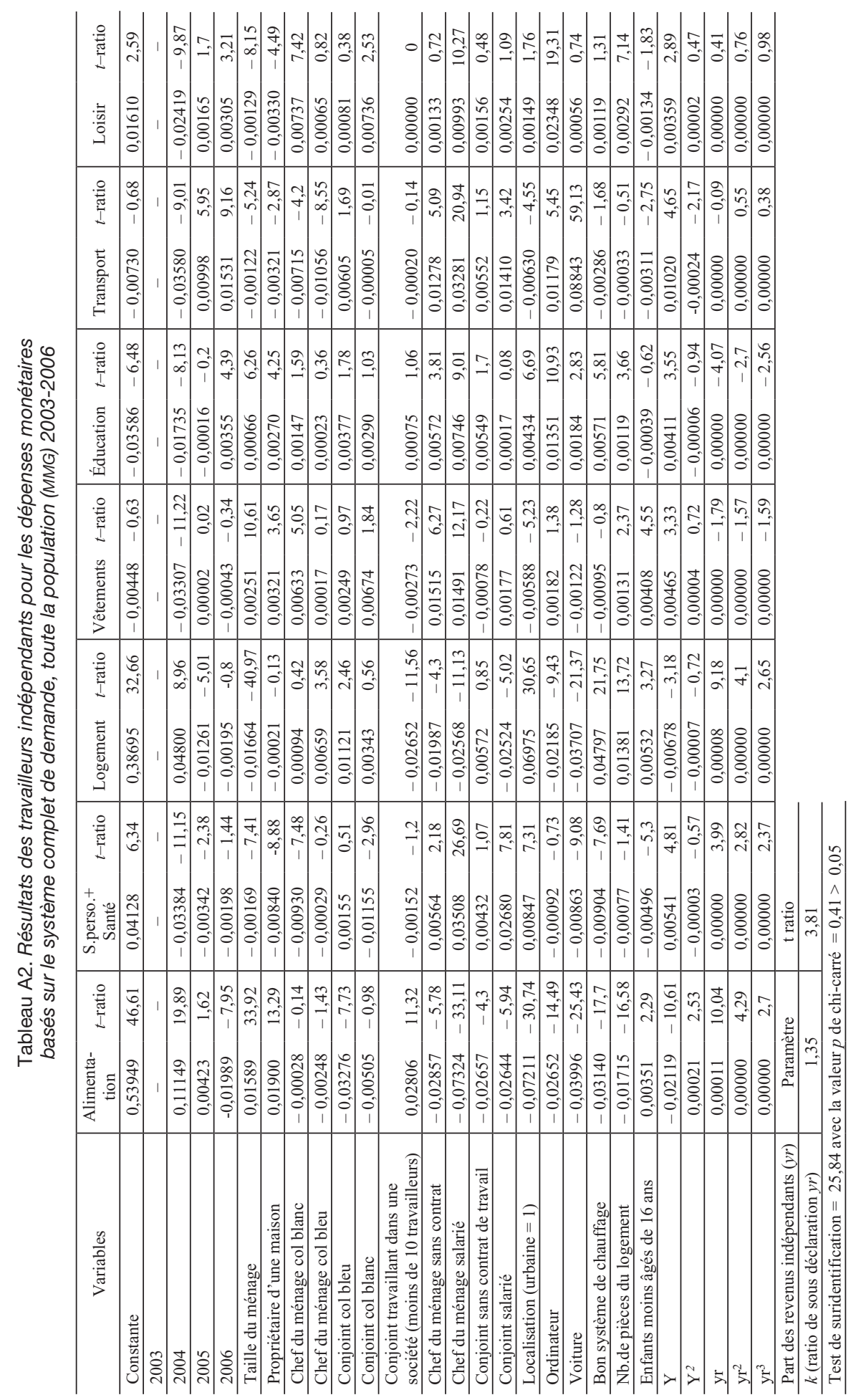


Tableau A3. Résultats d'estimation en deux étapes d'Heckman pour l'année 2006 Logarithme naturel du salaire mensuel

\begin{tabular}{|c|c|c|c|c|}
\hline \multirow{2}{*}{$\begin{array}{ll} & \text { Variables } \\
\text { Âge } & \end{array}$} & \multicolumn{2}{|c|}{ Homme } & \multicolumn{2}{|c|}{ Femme } \\
\hline & $\begin{array}{r}0,267 \\
(0,027)\end{array}$ & $* * *$ & $\begin{array}{r}0,405 \\
(0,098)\end{array}$ & $* * *$ \\
\hline Âge carré & $\begin{array}{l}-0,012 \\
(0,001)\end{array}$ & $* * *$ & $\begin{array}{l}-0,021 \\
(0,006)\end{array}$ & $* * *$ \\
\hline Enseignement primaire & $\begin{array}{r}0,484 \\
(0,028)\end{array}$ & $* * *$ & $\begin{array}{r}0,343 \\
(0,098)\end{array}$ & $* * *$ \\
\hline Enseignement secondaire & $\begin{array}{r}0,827 \\
(0,035)\end{array}$ & $* * *$ & $\begin{array}{l}1,465 \\
(0,128)\end{array}$ & $* * *$ \\
\hline Enseignement supérieur & $\begin{array}{r}1,193 \\
(0,040)\end{array}$ & $* * *$ & $\begin{array}{l}2,104 \\
(0,123)\end{array}$ & $* * *$ \\
\hline Localisation (urbaine) & $\begin{array}{r}0,250 \\
(0,017)\end{array}$ & $* * *$ & $\begin{array}{r}0,309 \\
(0,072)\end{array}$ & $* * *$ \\
\hline Couple & $\begin{array}{r}0,490 \\
(0,035)\end{array}$ & $* * *$ & $\begin{array}{r}5,300 \\
(0,050)\end{array}$ & $* * *$ \\
\hline Nombre d'enfants & $\begin{array}{r}0,713 \\
(0,010)\end{array}$ & $* * *$ & $\begin{array}{r}0,279 \\
(0,088)\end{array}$ & $* * *$ \\
\hline Taille des ménages & $\begin{array}{c}-0,196 \\
(0,041)\end{array}$ & $* * *$ & $\begin{array}{c}-0,186 \\
(0,017)\end{array}$ & $* * *$ \\
\hline Constante & $\begin{array}{c}4,651 \\
(0,118)\end{array}$ & $* * *$ & $\begin{array}{c}3,184 \\
(0,495)\end{array}$ & $* * *$ \\
\hline Termes d'erreur-corrélation rho & $\begin{array}{r}0,130 \\
(0,034)\end{array}$ & $* * *$ & $\begin{array}{l}-0,162 \\
(0,153)\end{array}$ & $*$ \\
\hline$\lambda$ & $\begin{array}{r}0,081 \\
(0,021) \\
\end{array}$ & $* * *$ & $\begin{array}{r}0,081 \\
(0,021) \\
\end{array}$ & $* * *$ \\
\hline Log de vraisemblance & -1231 & & -2976 & \\
\hline Nombre d'observations & 12875 & & 12033 & \\
\hline Nombre de travailleurs & 6094 & & 936 & \\
\hline
\end{tabular}


Tableau A4. Paramètres estimées de la sous-déclaration des revenus (2003-2006)

\begin{tabular}{cccc}
\cline { 2 - 4 } & Travailleurs & $\begin{array}{c}\text { Paramètre } \\
\mathrm{k}, \mathrm{v}(t \text {-ratio })\end{array}$ & IC (5\%) \\
\hline \multirow{3}{*}{$\begin{array}{c}\text { Dépenses } \\
\text { monétaires }\end{array}$} & $\begin{array}{c}\text { Travailleurs } \\
\text { indépendants }(k)\end{array}$ & $\begin{array}{c}1,35 \\
(3,81)\end{array}$ & {$[0,65 ; 2,04]$} \\
\cline { 2 - 4 } & $\begin{array}{c}\text { Travailleurs } \\
\text { salariés }(v)\end{array}$ & 0,25 & \\
& $\begin{array}{c}\text { Travailleurs } \\
\text { indépendants }(k)\end{array}$ & $1,35)$ & {$[0,13 ; 0,36]$} \\
\hline \multirow{2}{*}{$\begin{array}{c}\text { Dépenses } \\
\text { complètes }\end{array}$} & $\begin{array}{c}\text { Travailleurs } \\
\text { salariés }(\mathrm{v})\end{array}$ & 0,37 & {$[0,87 ; 2,40]$} \\
& & $(3,12)$ & {$[0,13 ; 0,60]$} \\
\hline
\end{tabular}

Tableau A5. Les parts des revenus des travailleurs indépendants et salariés (2003-2006)

(\% du PIB)

\begin{tabular}{l|c|c|c|c}
\cline { 2 - 5 } & 2003 & 2004 & 2005 & 2006 \\
\hline Travailleurs indépendants & 25,09 & 26,56 & 25,88 & 21,37 \\
\hline Travailleurs salariés & 50,70 & 54,50 & 57,00 & 58,90 \\
\hline \multicolumn{2}{l}{ Source : Institut des statistiques la Turquie (TUIK). }
\end{tabular}

Source : Institut des statistiques la Turquie (TUIK). 
Tableau A6. Mesures de la taille de l'économie informelle en Turquie

\begin{tabular}{|c|c|c|c|}
\hline Auteur & $\begin{array}{l}\text { Période/ } \\
\text { Année }\end{array}$ & Méthodes de mesure & $\begin{array}{l}\text { Taille de 1'économie } \\
\text { informelle (\% du PIB) }\end{array}$ \\
\hline $\begin{array}{l}\text { Hakioglu }(*) \\
\quad[1987] \\
{[1990]}\end{array}$ & $\begin{array}{l}1984 \\
1989\end{array}$ & Demande de monnaie & $\begin{array}{c}137,80 \\
18,00\end{array}$ \\
\hline $\begin{array}{c}\text { Derdiyok }(*) \\
{[1993]}\end{array}$ & $\begin{array}{l}1984 \\
1991\end{array}$ & $\begin{array}{c}\text { Approche monétaire } \\
\text { Audit fiscal }\end{array}$ & $\begin{array}{l}27,30 \\
46,94\end{array}$ \\
\hline $\begin{array}{c}\text { Altug }(*) \\
{[1993]}\end{array}$ & 1992 & Méthode de 1'emploi & 35 \\
\hline $\begin{array}{l}\text { Ozsoylu }(*) \\
\quad[1993]\end{array}$ & 1990 & $\begin{array}{c}\text { Approche du PNB } \\
\text { Ratio simple espèces/dépôts } \\
\text { Méthode des transactions monétaires }\end{array}$ & $\begin{array}{c}7,5 \\
11,50 \\
11,50\end{array}$ \\
\hline $\begin{array}{c}\text { Kasnakoglu }(*) \\
\text { [1993] }\end{array}$ & $\begin{array}{l}1968-1990 \\
1963-1990\end{array}$ & $\begin{array}{l}\text { Ratio espèces/dépôts } \\
\text { Demande de monnaie }\end{array}$ & $\begin{array}{c}-4,22-34,85 \\
0-22,55\end{array}$ \\
\hline $\begin{array}{c}\text { Temel et al. }(*) \\
\text { [1994] }\end{array}$ & $\begin{array}{l}1987-1992 \\
1984-1991 \\
1970-1992 \\
1970-1992 \\
1975-1992 \\
\end{array}$ & $\begin{array}{c}\text { Méthode de divergences } \\
\text { Audit fiscal } \\
\text { Ratio espèces/dépôts } \\
\text { Méthode des transactions monétaires } \\
\text { Demande de monnaie }\end{array}$ & $\begin{array}{c}1,48-3,61 \\
8,2-91,55 \\
0-26,26 \\
0-26,26 \\
-6,34-20,29\end{array}$ \\
\hline $\begin{array}{c}\text { Yayla }(*) \\
{[1995]}\end{array}$ & $1968-1993$ & $\begin{array}{c}\text { Ratio espèces/dépôts } \\
\text { Demande de monnaie } \\
\text { Méthode des transactions monétaires }\end{array}$ & $\begin{array}{c}-4,30-99,85 \\
0-42,09 \\
0-62,45\end{array}$ \\
\hline $\begin{array}{c}\text { Us } \\
{[2004]}\end{array}$ & $\begin{array}{l}1985-2002 \\
1978-2000 \\
1987-2003 \\
1987-2003\end{array}$ & $\begin{array}{c}\text { Audit fiscal } \\
\text { Approches des inputs physiques } \\
\text { Ratio espèces/dépôts } \\
\text { Demande de monnaie }\end{array}$ & $\begin{array}{c}26-184 \\
-1-33 \\
0-90 \\
3-12\end{array}$ \\
\hline $\begin{array}{l}\text { Baldemir et al. } \\
\text { [2009] }\end{array}$ & $1980-2003$ & MIMIC & $15,58-10,80$ \\
\hline $\begin{array}{c}\text { Akalin et Kesikoglu } \\
\text { [2007] }\end{array}$ & $\begin{array}{l}1975-2005 \\
1975-2005\end{array}$ & $\begin{array}{l}\text { Approche monétaire de base } \\
\text { Approche monétaire étendue }\end{array}$ & $\begin{array}{c}7-46 \\
17-139 \\
\end{array}$ \\
\hline $\begin{array}{c}\text { Schneider et Savasan } \\
{[2007]}\end{array}$ & $1999-2005$ & DYMIMIC & $31,1-35,1$ \\
\hline $\begin{array}{c}\text { Davutyan } \\
\text { [2008] }\end{array}$ & 2005 & Équation unique & 21 \\
\hline
\end{tabular}




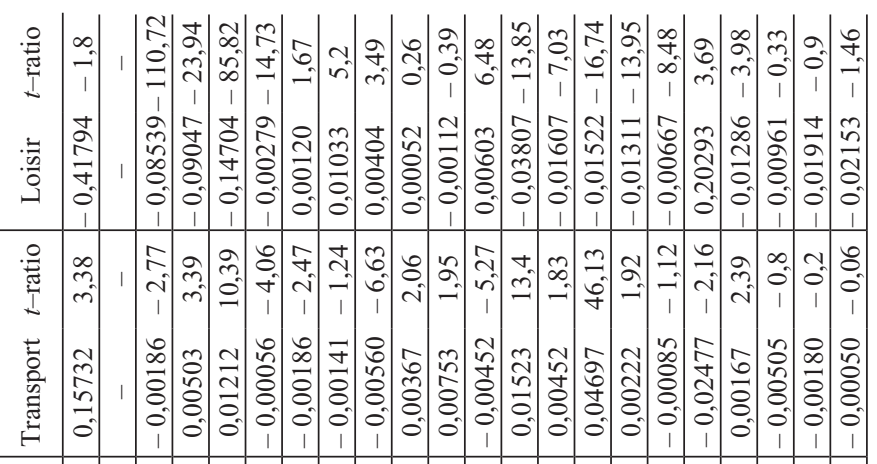

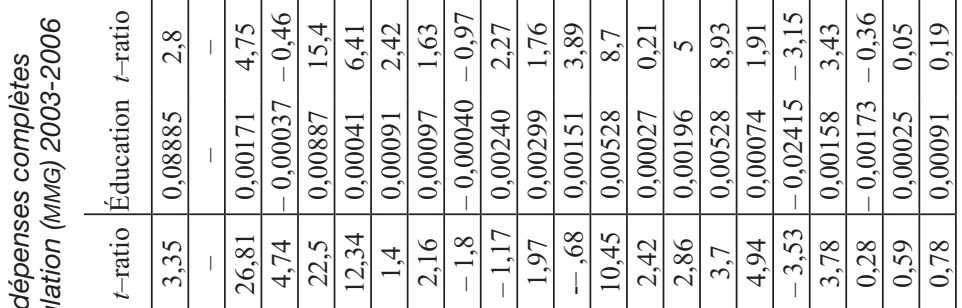

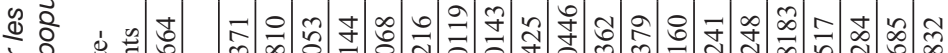
亏范

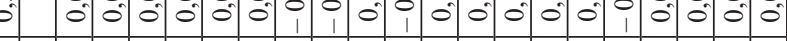

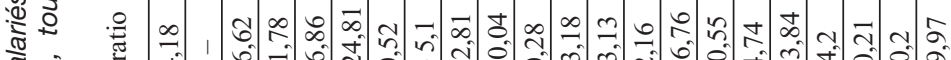

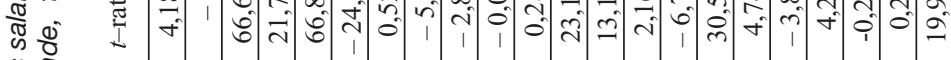
蛋

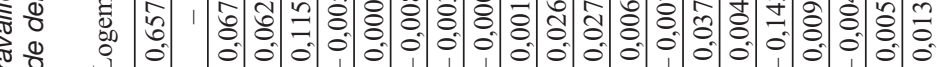

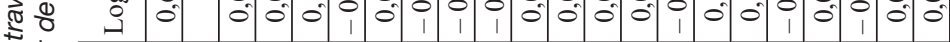

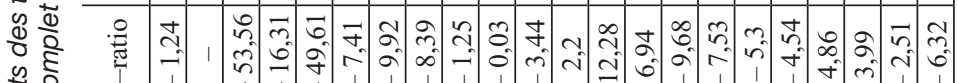
స్

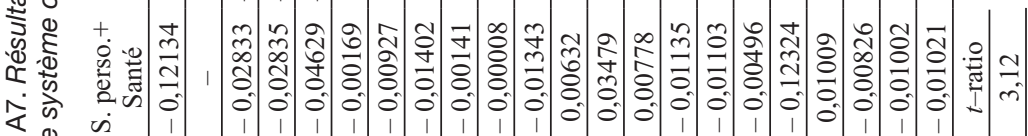

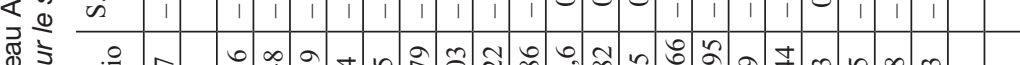

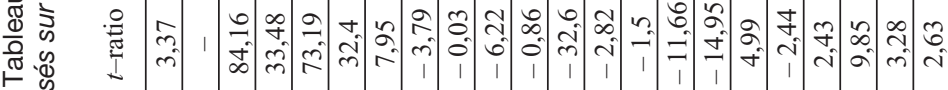

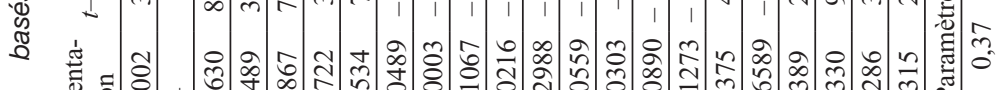

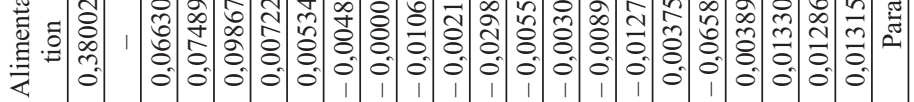

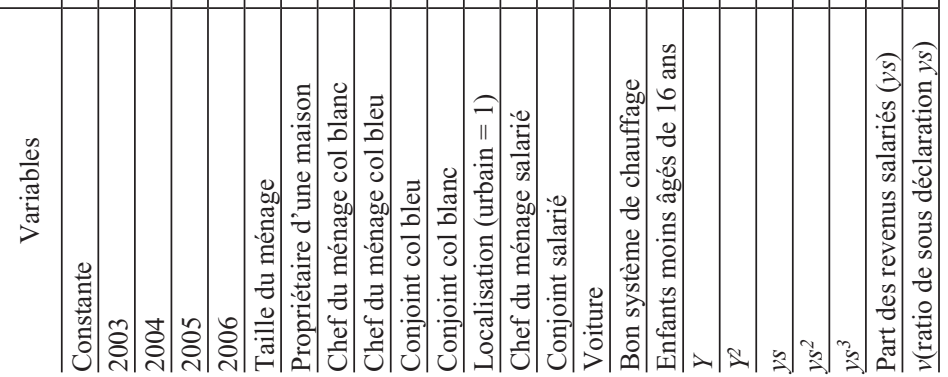




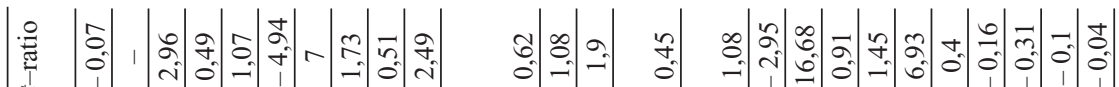

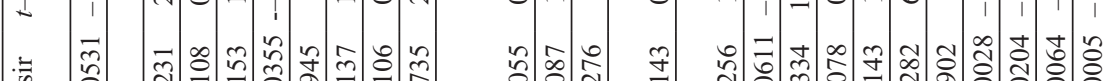

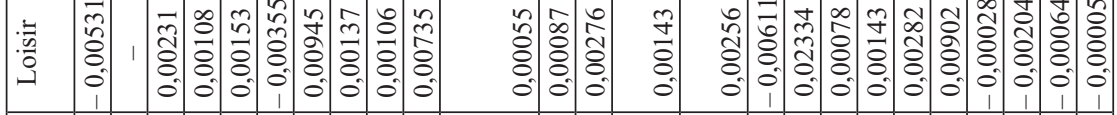

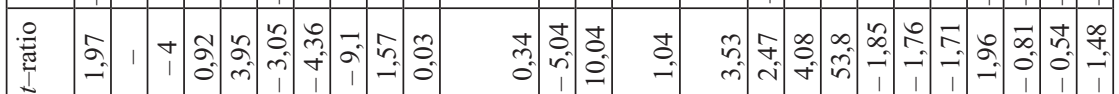

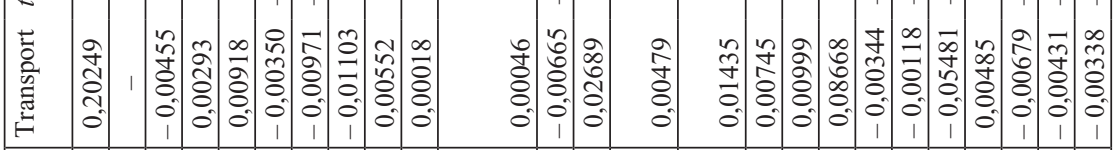

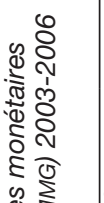

.류

I 0 o

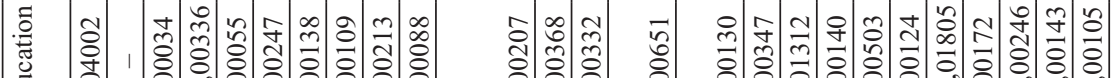

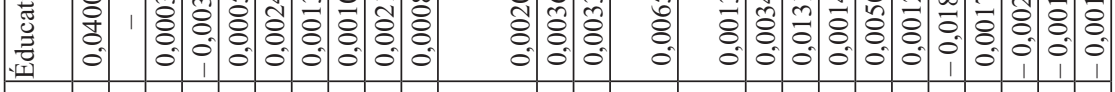

幽

(1)

寻

$\frac{0}{0} \frac{\pi}{\frac{\pi}{0}}$

\&

$\lesssim \frac{2}{2}$

20

巴)

$\frac{\pi}{2}+$

के $\frac{0}{2}$

๗ ฐิ

(⿻)

ते $\frac{0}{0}$

ป d $\frac{1}{2}$

赵

$\stackrel{\pi}{5} \stackrel{0}{5}$

这

$\infty$ के

\&

गัญ

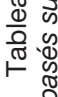

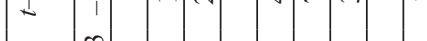

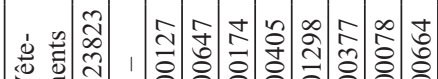

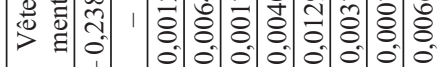

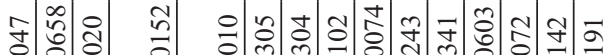

局

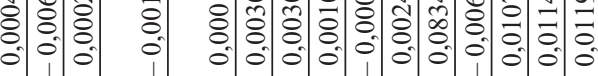

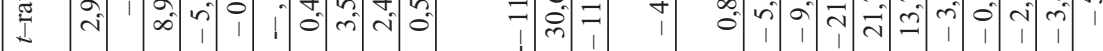

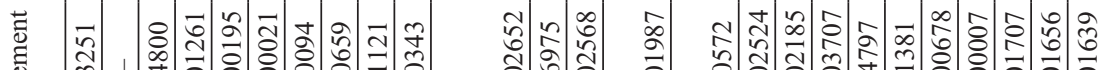

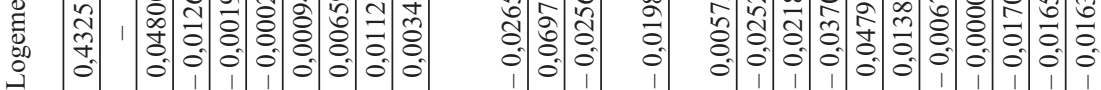

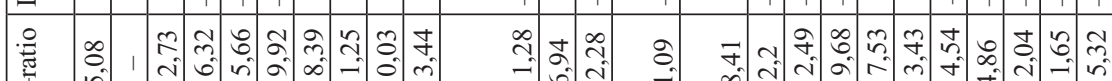

नी

+.

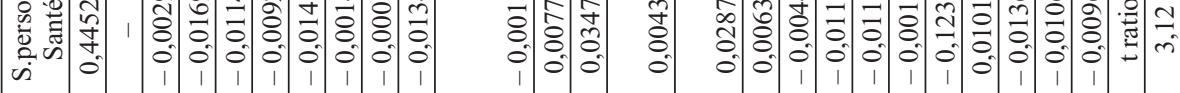

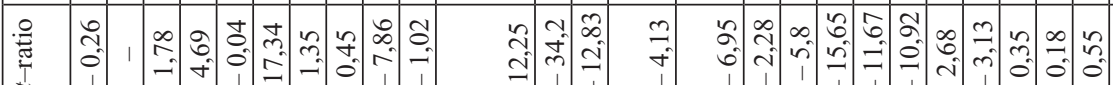

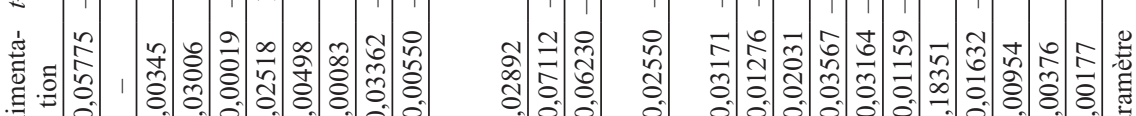

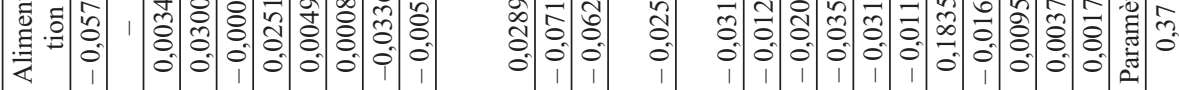

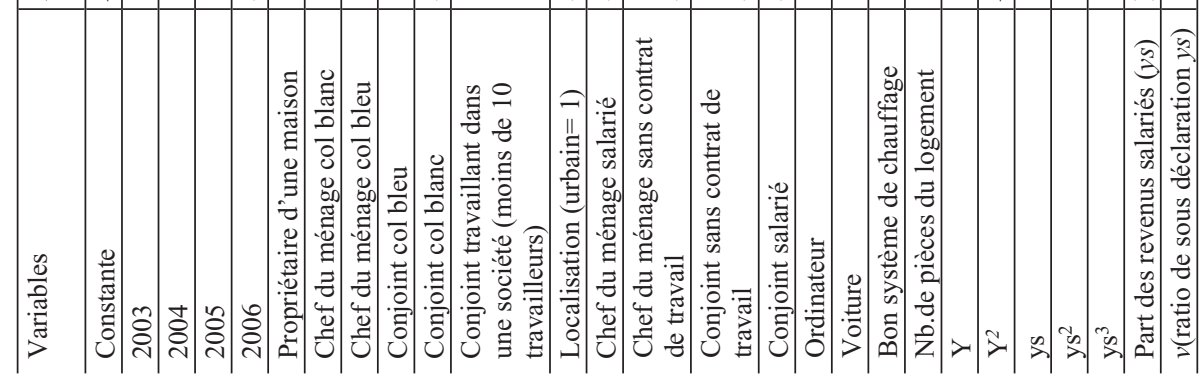




\section{ANNEXE II}

\section{LES REVENUS DES ACTIVITÉS INDÉPENDANTES REDRESSÉS \\ POUR L'ESTIMATION DES REVENUS SALARIAUX NON DÉCLARÉS}

Une fois l'équation (7) estimée, les paramètres obtenus des courbes d'Engel sont utilisées pour le calcul des vrais revenus d'indépendants comme $\mathrm{Y}_{r}^{*}=\bar{\theta}_{r} \mathrm{Y}_{r}$ où $\mathrm{Y}_{r}^{*}$ est le revenu ajusté d'indépendants obtenu par la multiplication des revenus déclarés des indépendants $\mathrm{Y}_{r}$ par $\bar{\theta}_{r}$. Ce paramètre s'écrit en sommation sur les catégories de dépenses :

$$
\bar{\theta}_{r}=\frac{1}{n} \sum\left(\frac{10^{\widehat{R}}-\sum_{m=a, s, r} \hat{\theta}_{m} y_{m}}{y_{r}}\right) .
$$

Cette équation est dérivée de l'équation (7). $\theta_{r}$ est calculé pour chaque catégorie de consommation en utilisant les paramètres estimés du système complet de demande (équation (7)). $\bar{\theta}_{r}$ est la moyenne arithmétique de $\theta_{r}$ pour $n$ catégories de consommation ${ }^{17}$.

17. Dans le cadre du modèle quadratique, $\widehat{\mathrm{R}}$ se définit
$\widehat{\mathrm{R}}=-\hat{\beta}_{i}-2 \hat{\delta}_{i}+\sqrt{\left(\hat{\beta}_{i}+2 \hat{\delta}_{i}\right)^{2}-4 \hat{\delta}_{i}\left(\hat{\beta}_{i} \ln \mathrm{Y}_{h}\right)^{2}-\left(\hat{\alpha}_{i}+\sum_{j} \hat{\alpha}_{j} Z_{j h}+\sum_{n=1}^{3} \hat{\lambda}_{i n}\left(y_{r, s}\right)^{n}+\sum_{t} \hat{\alpha}_{i} \mathrm{~A}_{t}\right)}$. est la variable muette de l'année. $\widehat{\mathrm{R}}$ est une équation discriminante ; par conséquent, seule la racine positive est choisie. 
